

Antropología Social

\title{
Pueblos indígenas y Estado en torno a la conservación de la naturaleza: el caso del territorio de la comunidad Potae Napocna Navogoh y las tierras del Parque Nacional Río Pilcomayo
}

Indigenous People and the State around the Conservation of Nature: the Case of the Territory of the Potae Napocna Navogoh Community and the Lands of the Pilcomayo River National Park

Valeria Iñigo Carrera*

*Universidad Nacional de Río Negro - Consejo Nacional de Investigaciones Científicas y Técnicas. Instituto de Investigaciones en Diversidad Cultural y Procesos de Cambio. Argentina. E-mail: v.inigocarrera@conicet.gov.ar

\begin{abstract}
Resumen
En el presente trabajo nos proponemos abordar la pregunta por la relación entre los pueblos indígenas y la conservación de la naturaleza, a propósito de la superposición entre el territorio de la comunidad Potae Napocna Navogoh y las tierras del Parque Nacional Río Pilcomayo (provincia de Formosa, Argentina). En particular, nuestro objeto es clarificar el vínculo entre el desarrollo de áreas naturales protegidas y la producción de una subjetividad indígena esencialmente diferencial. Para ello, comenzamos por examinar la tensión que se manifiesta al interior de la política seguida por los Estados (nacional y provincial) entre la valorización del capital y aquella conservación. Luego, caracterizamos las maneras en que los pueblos indígenas son producidos por los mencionados Estados en el escenario político provincial. Finalmente, estudiamos el caso del conflicto producto de la mencionada superposición, a partir del análisis de una reunión sostenida en el año 2007 entre los qom y distintos agentes del Estado.
\end{abstract}

Palabras claves: Pueblos indígenas; Estado; Territorio; Conservación de la naturaleza; Formosa

\begin{abstract}
In this work we aim to address the question on the relationship between indigenous peoples and nature conservation, regarding the overlap between the territory of the Potae Napocna Navogoh community and the lands of the Pilcomayo River National Park (province of Formosa, Argentina). In particular, our purpose is to clarify the link between the development of protected natural areas and the production of an essentially differential indigenous subjectivity. To do this, we begin by examining the tension that manifests itself within the policy followed by the states (national and provincial) between the valorisation of capital and nature conservation. Then, we characterize the ways in which indigenous peoples are produced by the mentioned states in the provincial political scene. Finally, we study the case of the conflict resulting from the above mentioned overlap, based on the analysis of a meeting held in 2007 between the qom and various state agents.
\end{abstract}

Key words: Indigenous Peoples; State; Territory; Nature Conservation; Formosa

La conflictividad resultante de la superposición entre unidades de conservación de la naturaleza y territorios indígenas ocupados y/o reclamados constituye una realidad recurrente en Latinoamérica. Esto es así, a pesar de que: a) desde fines de la década de 1980 ha tenido lugar un proceso de cuestionamiento al modelo de conservación sobre el que se fundó a lo largo del siglo XX la creación de las áreas protegidas en la región -modelo que excluía a las poblaciones humanas de esos espacios en tanto consideraba su presencia como una amenaza para la conservación-; b) sobre la base de ese cuestionamiento, se han introducido cambios en las políticas y legislaciones sobre áreas protegidas tendientes a la construcción de nuevos paradigmas en la relación entre tales áreas y los pueblos indígenas, basados en el reconocimiento de derechos especiales sobre sus tierras, territorios y recursos en ellos contenidos (Aylwin, 2011).

La Argentina no es ajena a estos procesos. Si bien el Estado ha configurado un marco jurídico específico para los pueblos indígenas, que parece contener aquel cambio de paradigma, es indudable que las reivindicaciones y demandas indígenas en relación con el territorio en general y con aquellas tierras comprendidas en áreas 
de conservación de la naturaleza en particular aún encuentran un lugar destacado en el conflicto social. ${ }^{1}$ En el noreste de la provincia de Formosa, el conflicto es entre el Parque Nacional Río Pilcomayo (PNRP) y la comunidad qom Potae Napocna Navogoh. También conocida como La Primavera, esta última está ubicada a ambos márgenes de la ruta nacional $\mathrm{N}^{\circ} 86$, de manera cercana al río Pilcomayo (límite, allí, con la República del Paraguay) y distante unos $140 \mathrm{~km}$ de la capital formoseña, en el departamento Pilcomayo. El área protegida, por su parte, fue creada sobre el territorio históricamente ocupado y hoy reclamado por los qom. Son su desposesión y la restricción en el acceso a los recursos naturales de la zona -sólo por enumerar las expresiones inmediatamente materiales- las que se encuentran en la base del conflicto entre la comunidad y la administración del parque.

En el marco de este tipo de conflictos, un argumento que aparece de manera frecuente, ya sea para fundar la mencionada política de reconocimiento hacia los pueblos indígenas o bien la reivindicación de su derecho al territorio en el marco de esa política, es el carácter estrecho y armónico de su vínculo con la naturaleza, y el consecuente carácter estratégico del rol de esos pueblos en su conservación. Sobre esta base, en este trabajo nos convoca la cuestión de la relación entre el desarrollo de áreas naturales protegidas y la producción de una subjetividad indígena esencialmente diferencial.

En el ámbito de la antropología argentina, son diversos los trabajos que, de manera reciente, han abordado esa cuestión. En su generalidad (Carenzo y Trentini, 2014; Ferrero, 2014; Papalia, 2012; Trentini, 2016), coinciden en sostener, en consonancia con los desarrollos producidos en y/o sobre Latinoamérica, que en el marco de los modelos contemporáneos de manejo de las áreas protegidas y de la integración de las poblaciones locales que las habitan a ese manejo sobre la base de la recuperación y valorización de sus prácticas y saberes, se construye la subjetividad indígena en términos esencialistas bajo la forma de las figuras del "buen salvaje ecológico" (Redford, 1991), el "eco-indio" (Conklin y Graham, 1995) o el "nativo ecológico" (Ulloa, 2001). En estos análisis, se atribuye la construcción en esos términos a un modelo de gobernanza neoliberal de la naturaleza que supedita el reconocimiento a los pueblos indígenas de derechos sobre el territorio a su capacidad para encarnar ese "esencialismo verde" (Carenzo y Trentini, 2014; Trentini, 2015); y, en sintonía con lo anterior, a un modelo que a la par de implicar formas de control y disciplinamiento

\footnotetext{
1 Tanto es así que, en noviembre de 2017, la recuperación territorial realizada por el lof Lafken Winkul Mapu -la de un territorio que ha quedado comprendido dentro de los límites del Parque Nacional Nahuel Huapi (PNNH), en la Patagonia argentina- ha resultado, tras la denuncia efectuada por la Administración de Parques Nacionales (APN), en la acción represiva del Estado nacional a través del desalojo de algunos de sus miembros, la detención de otros y el asesinato de uno de ellos a manos de las fuerzas de seguridad (Río Negro, 25/11/2017)
}

de la acción política de las poblaciones locales (entre otras, las indígenas) crea nuevas posibilidades políticas para ellas (Ferrero, 2014; Papalia, 2012). Del otro lado, esto es, en tanto se trata de una construcción que esos mismos análisis evidencian propia no sólo de las políticas de conservación sino también de las organizaciones de pueblos indígenas, la atribuyen a una estrategia política de estas últimas en el marco de la reivindicación de sus derechos territoriales (Carpinetti, 2007; Ferrero et al., 2015; Schmidt, 2013; Trentini, 2015).

A propósito del conflicto entre la comunidad Potae Napocna Navogoh y el PNRP, con este trabajo nos proponemos aportar a las explicaciones contenidas en la literatura especializada producida en el ámbito académico local a partir de un abordaje materialista del vínculo entre el desarrollo de áreas naturales protegidas y la producción de una subjetividad indígena esencialmente diferencial -puesto que, adelantamos aquí, son éstos los términos en los que se construye, en el caso analizado también, esa subjetividad-. En otras palabras, procuramos aproximarnos a ese vínculo anclándolo en la organización de la materialidad del proceso de vida social a través de la acumulación de capital (J. Iñigo Carrera, 2012). ¿Cuál es la necesidad que se realiza bajo la forma específica de una subjetividad indígena construida en aquellos términos en el marco de las actuales maneras de conservación de la naturaleza? Desde este punto de vista, esa subjetividad indígena no es sino una forma concreta, necesaria y portadora de múltiples determinaciones, de cuya especificidad debemos dar cuenta partiendo de la relación social general que rige la organización capitalista de la producción social, hasta alcanzar la mediación que determina dicha especificidad por su condición de población indígena (J. Iñigo Carrera y V. Iñigo Carrera, 2017).

Sobre esta base, hemos estructurado el trabajo del siguiente modo. Dedicamos los dos apartados que siguen a la política de Estado, en primer lugar, en relación con la valorización del capital y la conservación de la naturaleza y, en segundo lugar, en relación con la producción de una ciudadanía diferencial para los pueblos indígenas. Tras presentar algunos antecedentes del referido conflicto, abordamos su estudio a partir del análisis de una reunión sostenida en el mes de mayo del año 2007 entre los qom, funcionarios de la APN, del mencionado parque nacional y de la Dirección de Pueblos Originarios y Recursos Naturales (DPOyRN) de la Secretaría de Ambiente y Desarrollo Sustentable de la Nación (SAyDS). Este evento, de carácter extraordinario, tuvo lugar en los momentos iniciales de un proceso de movilización política protagonizado por los qom de Potae Napocna Navogoh que, de ahí en más, ganaría en intensidad y conflictividad; a la vez, puso en acto una serie de prácticas y sentidos significativos a la hora de encarar la cuestión de la relación entre los pueblos indígenas y la conservación de la naturaleza. Su análisis se basa no sólo 
en la observación directa y el registro de esa reunión sino también en la realización de entrevistas en profundidad y la lectura de documentos referidos al PNRP. ${ }^{2}$ Nuestro interés es dar cuenta tanto de las prácticas actuadas por los qom en relación con la apropiación y transformación de la naturaleza como de los sentidos que les atribuyen en diálogo con las maneras en que, desde el Estado, se (re)presenta esa relación.

\section{La política de Estado I: entre la valorización del capital y la conservación de la naturaleza}

Desde los inicios del desarrollo de su curso histórico, el avance del capital encontró distintas expresiones en la provincia de Formosa: los obrajes madereros, los ingenios azucareros y las plantaciones algodoneras. Llevadas adelante en establecimientos de baja inversión tecnológica y productividad, la producción provincial de tanino y caña de azúcar fueron históricamente marginales en relación con la nacional. ${ }^{3}$ Por su parte, la agricultura de secano tuvo poca significación en la totalidad del panorama productivo nacional e incluso formoseño hasta la década de 1930. Entonces, en el marco, primero, de una demanda externa favorable y, luego, de una demanda interna en expansión, se abrió paso de manera decidida la producción centrada en el algodón. Constituido, de ahí en más, en el principal cultivo de la provincia, su producción se realizó, desde sus inicios, en unidades productivas pequeñas y medianas, y fue impulsada fuertemente por el Estado a través de la asistencia técnica y financiera, la comercialización y la instalación de desmotadoras oficiales y de escuelas de clasificadores del textil (Guy, 2000).

El desarrollo de los distintos sistemas productivos tuvo por condición de posibilidad la disponibilidad de mano de obra indígena y su transformación en trabajadores productivos para el capital. La producción de algodón fue la que absorbió en mayor grado aquella mano de obra. En su constitución en pequeños productores de algodón en bruto y trabajadores asalariados estacionales en la carpida y cosecha del cultivo, el Estado se consolidó en su rol de promotor: la orientación hacia la labor agrícola, el disciplinamiento en la organización de los procesos de trabajo, el adiestramiento en la comercialización de

\footnotetext{
2 Fueron consultados los materiales (legislación, expedientes, fichas de pobladores) contenidos en la Biblioteca Central y Centro de Documentación Perito Francisco P. Moreno y en el Programa Pobladores y Comunidades de la Dirección Nacional de Conservación de Áreas Protegidas (APN).

3 Los establecimientos madereros se repartieron sobre los ríos Pilcomayo, Paraguay y Bermejo y sobre distintos riachos del borde oriental del Territorio; en 1905, se instaló en la ciudad de Formosa la primera fábrica de extracto tánico, dando comienzo a una intensiva explotación del quebracho colorado de los montes formoseños (Brodersohn y Slutzky, 1975). Por su parte, los dos principales ingenios azucareros fueron establecidos de manera cercana a la planta urbana de la ciudad de Formosa (Ingenio La Teutonia) y a la desembocadura del Pilcomayo (Ingenio Santa Elena); instalados a fines de la década de 1880, su permanencia apenas superará la entrada al nuevo siglo (Prieto, 2015).
}

su producción, la administración de su subsistencia y su instrucción escolar, moral y religiosa fueron propósitos encarnados por las reducciones estatales y por las misiones religiosas que se instalaron en el Territorio Nacional de Formosa desde comienzos del siglo XX. Aún hoy, el Estado se mantiene en ese rol a través del despliegue de mecanismos similares a los de antaño: la entrega de semillas, implementos agrícolas y alimentos (V. Iñigo Carrera, 2014).

En las últimas décadas, la escala de la producción algodonera formoseña fue objeto de violentas oscilaciones de una campaña agrícola a otra. Por sobre estos movimientos circunstanciales se evidencia una tendencia decreciente en aquella escala. Así, en la década de 1970, la producción formoseña alcanzó su pico histórico, llegando a representar el $16 \%$ del total nacional. Pero a partir de entonces su escala entró en un proceso de continua contracción: en la primera mitad de la década de 2000, representó, en promedio, menos del 3\% de la producción nacional (J. Iñigo Carrera y V. Iñigo Carrera, 2017). A diferencia de lo que sucede en otras provincias del Chaco argentino, el avance de la soja sobre la superficie agrícola disponible, si bien es de una tendencia manifiestamente creciente, se revela también errático y moderado. Frente al anquilosamiento de la actividad agrícola, la cría de ganado vacuno se expande sobre la base de la reconversión de la estructura productiva en función del desarrollo de una ganadería modernizada (con la introducción de tecnología mecánica y sanitaria y de pasturas artificiales), protagonizado por aquellas explotaciones que cuentan con alta inversión de capital. Mucho tuvo que ver en esa modernización el Estado formoseño a través de la privatización de la tierra a manos de terratenientes locales y empresas extra-provinciales, en particular, la de la tierra comprendida en la porción oriental de la provincia (Sapkus, 2014).

En el marco de este proceso de promoción del capital más concentrado fundado sobre la reversión de plusvalía a nuevo capital, el incremento de la infraestructura vial, de manejo de los recursos hídricos, energética y urbana aparece como una problemática central. En tanto se trata de una provincia históricamente pobre en materia de concreción de obras básicas de infraestructura, no es novedoso el planteo de la necesidad de su modernización con vistas a generar condiciones propicias para la inversión de capital. Sin embargo, su inserción en distintos procesos de integración regional, y su participación en los instrumentos de desarrollo de las economías nacionales y/o regionales que los componen, le otorgó un renovado dinamismo a aquella generación a partir de los años $1990 .^{4}$

\footnotetext{
${ }^{4}$ Nos referimos al Mercado Común del Sur, a la Zona de Integración del Centro Oeste de América del Sur, a la Iniciativa para la Integración de la Infraestructura Regional Suramericana y al Consejo Suramericano de Infraestructura y Planeamiento; todos ellos, mecanismos institucionales dirigidos a alcanzar la integración regional a través de la coordinación de políticas macroeconómicas y sectoriales, y la planificación e
} 
Dicha inserción, en tanto valorización de los capitales industriales aplicados a diversas actividades productivas, no es sino una de las maneras en que el Estado se constituye en representante político del conjunto de los capitales de la sociedad (J. Iñigo Carrera, 2013). ${ }^{5}$ Otra de las maneras, que en apariencia resulta contradictoria, es el desarrollo por parte de los Estados provincial y nacional, y de organismos internacionales, de una política de conservación de la naturaleza, que se materializa en la creación de reservas naturales, áreas naturales protegidas, reservas de biósfera. ¿Por qué decimos que esta última resulta contradictoria sólo en apariencia? Ya en la primera mitad del siglo XX, con la creación en 1934 del PNNH, estas áreas de conservación no fueron entendidas únicamente como unas de preservación del ambiente sino que estaban asociadas al fomento de nuevas formas de industria como la turística (Navarro Floria, 2007; Pérez y Delrio, 2019) y al ejercicio de la soberanía territorial en espacios fronterizos (Navarro Floria, 2008; Trentini, 2016).

Por cierto, en notas elevadas en 1944 por el gobernador del Territorio Nacional de Formosa, Coronel Conrado Sztyrle, a la Dirección de Parques Nacionales y Turismo, aquél solicitaba se contemplara la posibilidad de crear un parque nacional en ese Territorio por la necesidad de disponer de un área de conservación de la naturaleza en su estado primitivo "que pueda ofrecer a las generaciones actuales y futuras una expresión real de las características integrales del Territorio, en lo que se refiere en sus aspectos físicos y especialmente en materia de fauna y flora" (Ministerio de Agricultura, Dirección de Parques Nacionales y Turismo, Expediente $N^{\circ} 1919 / 44$, fs. 2). Pero también el entonces gobernador hacía referencia a la necesidad de construir, de manera simultánea, un hotel de turismo en la ciudad de Formosa "a fin de satisfacer un legítimo anhelo de los viajeros propios y extranjeros, que desean junto con el noble afán de visitar nuestro suelo, comprobar la pujanza de sus riquezas y las halagüeñas perspectivas de su porvenir" (Ministerio de Agricultura de la Nación, Dirección de Parques Nacionales y Turismo, Expediente $N^{\circ} 1919 / 44$, fs. 4). Por su parte, el entonces presidente de la Comisión Nacional de Zonas de Seguridad, General de Brigada Enrique Jáuregui, en nota elevada en 1946 al Administrador General de Parques Nacionales y Turismo, expresaba su "más entusiasta y decidido deseo de que la iniciativa del señor Gobernador sea cuanto antes llevada a la práctica" teniendo en cuenta "el problema de nuestra seguridad fronteriza desde el punto de vista de su ocupación en tiempos de paz" (Ministerio de Agricultura de la Nación, Dirección de

implementación de programas y proyectos de modernización de la infraestructura regional.

5 Según este autor, la especificidad histórica del Estado como representante político del capital total de la sociedad encuentra su fundamento en la doble necesidad de ese capital de tener su propio representante político en la lucha de clases y de que ésta tome la apariencia de su contrario. Esta doble necesidad toma la forma concreta de la relación de ciudadanía del Estado (J. Iñigo Carrera, 2013).
Parques Nacionales y Turismo, Expediente No 1919/44, fs. 22). Aquí también, a las razones de conservación de la naturaleza se sumaban las de promoción industrial y defensa nacional.

En esas mismas notas se hacía referencia a la necesidad de declarar de utilidad pública y expropiar campos de propiedad privada, puesto que la extensa superficie entonces definida para el futuro parque comprendía en su interior estancias dedicadas a la ganadería y lotes de la Colonia Pastoril Laguna Blanca. Por cierto, al momento de su creación en 1951 a través de la Ley $N^{\circ}$ 14.073, el PNRP contaba con una superficie aproximada de 285.000 ha. Numerosos pobladores criollos, de origen paraguayo en un alto porcentaje, pero también español e italiano, dedicados al cultivo del algodón y a la ganadería extensiva, con una tenencia precaria de la tierra, se encontraban asimismo asentados en el área destinada a conservar. Entonces, el parque se erigió sobre su desalojo y reubicación. Sobre la base de la Ley $N^{\circ} 12.103$ de Creación de la Dirección de Parques Nacionales, en 1969 se suscribió un convenio entre la APN y la provincia por el que esta última se comprometía a reubicar a los pobladores desalojados.

Asimismo, por Ley $N^{\circ} 17.915$, sancionada en 1968, se redujo la superficie inicial del PNRP a la extensión actual de 51.889 ha, reincorporándose la superficie restante al dominio de la provincia de Formosa. ${ }^{6}$ La ley establecía que

"la región este del Parque ha sufrido una profunda transformación en su naturaleza, por los numerosos pobladores que allí se han radicado dedicando la tierra a cultivos, explotación ganadera, llegándose hasta a formar núcleos urbanos. En tales condiciones resulta difícil recuperar esas áreas para el fin a que fueron destinadas al crearse el referido Parque, por lo que se estima procedente su exclusión, facilitando así a la Provincia regularizar la situación hoy incierta de esos pobladores".

En verdad, se trataba de tierras de interés para el gobierno provincial por las condiciones del suelo y las potencialidades resultantes para la producción agrícola y ganadera. Como decía el entonces gobernador de la provincia, Augusto Sosa Laprida, en nota de 1967 dirigida al presidente de la Dirección Nacional de Parques, "dicha zona es una de las más importantes de esta Provincia en lo que respecta a su valor productivo y económico, y su ubicación dentro del Parque Nacional trae consigo una serie de inconvenientes jurídicos, técnicos y económicos

\footnotetext{
${ }^{6}$ La desafectación de más de 200.000 ha entra en clara contradicción con el principio de no regresividad o de progresividad consagrado en la hoy vigente Ley General del Ambiente N² 25.675 (sancionada en 2002) respecto de los niveles de protección ya alcanzados. Este principio implica, por un lado, la obligación de que los objetivos ambientales sean logrados en forma gradual y, por otro, la imposibilidad de la disminución de la protección del ambiente.
} 
que repercuten contra su desarrollo" (Dirección General de Parques Nacionales, Expediente No 5179/67: fs. 2). En otras palabras, su inclusión dentro de los límites originales del parque no hacía sino entorpecer el mentado desarrollo económico de la zona así como las posibilidades de reproducción de quienes, según el entonces Director de Colonización y Tierras Fiscales, habían abierto paso al progreso, combatiendo con la naturaleza y transformándola en campos productivos (Ministerio del Interior de la Nación, Expediente N 85/72: fs. 10-11). Los pedidos en el sentido de achicar la superficie del PNRP habían comenzado en 1956 y continuarían años más tarde de su reducción. ${ }^{7}$ Sin embargo, aun después de esta última, el parque continuó contando, en su jurisdicción, con la presencia de numerosos pobladores criollos, dedicados a la explotación ganadera. Recién en 1995 se levantaron sus puestos, desalojándose la totalidad del ganado vacuno (Entrocassi Fassinato y Espínola, 2006).

Pero no eran sólo criollos los pobladores que habitaban el área destinada a conservar: en su interior, quedó gran parte del territorio ocupado históricamente por los qom de Potae Napocna Navogoh -una ocupación que se remonta en mucho a la realizada por los primeros-. En este sentido, tras la aparente contradicción entre la valorización del capital y la conservación de la naturaleza -expresada en la sustracción por parte del Estado de las tierras en cuestión a la primera con fines vinculados a la segunda- se esconde la (re)producción de una porción de la población trabajadora (la indígena) con, veremos, atributos productivos mutilados. ${ }^{8}$

\section{La política de Estado II: la producción de una ciudadanía diferencial para los pueblos indígenas}

Durante largo tiempo, la población indígena se encontró por fuera de la relación política general de ciudadanía del Estado -que, en términos formales, es la expresión plena de la subjetividad política en la organización capitalista de la producción social-. ${ }^{9}$ Por cierto, los indígenas no eran considerados ciudadanos libres e iguales con acceso

\footnotetext{
${ }^{7}$ Se llegó, incluso, a solicitar su eliminación. En 1972, Sosa Laprida fundaba su solicitud en que: "La propiedad y disposición de dichas tierras por la Provincia satisfará demandas de agricultores y ganaderos, y radicación de industrias que [...] darán a esa zona una vivencia extraordinaria, transformando un espacio vacío e improductivo, en una zona de frontera, rica en realizaciones de todo orden" (Ministerio del Interior de la Nación, Expediente No 85/72: fs. 42-43).

${ }^{8}$ La relación entre la creación de unidades de conservación de la naturaleza y la (re)creación de las condiciones para la acumulación de capital -a través del cercamiento y la desposesión de la tierra y los recursos naturales, de la exclusión de las poblaciones locales y su incorporación al trabajo asalariado con vistas a alcanzar la reproducción social de su vida, de la promoción del turismo basado en la naturaleza, entre otros mecanismos- ha sido señalada por diversos autores (Brockington y Duffy, 2010; Kelly, 2011; entre otros). Se trata de un vínculo que -pese a que no siempre resulta obvio o inmediatoposibilita un mejor entendimiento de, en nuestro caso, la trayectoria (los vaivenes y las tensiones que encierra) del PNRP.

9 Este apartado es una versión abreviada de desarrollos contenidos en trabajos anteriores (V. Iñigo Carrera, 2011, 2015).
}

pleno a los derechos conferidos a los restantes habitantes del suelo argentino. Recién durante los años del primer peronismo (1946-1955), se procedió a su inscripción en el Registro Civil y a la entrega de documentos de identidad (Gordillo, 2006) -a la vez, se avanzó en la provincialización de los Territorios Nacionales (Formosa pasó a ser provincia en 1955), poniéndosele fin a las restricciones en el ejercicio de la ciudadanía política para sus habitantes (Ruffini, 2005)-. Entonces, fue su condición general de trabajadores la que se instituyó en la base sobre la que fue reconocida su condición general de ciudadanos del Estado argentino. En ese momento, la acumulación del capital necesitaba de la producción de obreros relativamente universales dentro de cada ámbito nacional -por más mutilados que se encontraran sus atributos productivosportadores de derechos igualitarios como ciudadanos del Estado nacional. Sin embargo, a partir de fines de los años setenta, la tendencia hacia la universalidad nacional con que se reproducía el obrero deja lugar a una diferenciación creciente fundada en su calificación que es, también, una diferenciación de ciudadanía al interior de la clase obrera (J. Iñigo Carrera, 2013).

Es así como, desde fines de la década de 1980, bajo el revestimiento de una ideología del multiculturalismo propia del capital en su fase neoliberal, que promueve el reconocimiento de derechos particulares por sectores de población al interior de cada ámbito nacional con base en la exaltación de su diferencia cultural, emergió una nueva concepción de ciudadanía para los pueblos indígenas. En ella, la premisa de la universalidad se ve constantemente tensionada. Dice Álvaro Bello (2009) que, en Latinoamérica, se trató de una forma de administrar las diferencias culturales al interior de los Estados nacionales sobre la base de su reconocimiento y promoción -esto es, de manera distinta a la disolución de la diferencia cultural propia de las políticas de integración del indigenismo de Estado-.

Sobre la base del reconocimiento de la multietnicidad y el pluriculturalismo, el Estado argentino se ha constituido en productor de una legislación propia y suscriptor de una serie de declaraciones y convenios internacionales. Ha dado forma así a un marco jurídico específico para los indígenas, con base en su carácter de sujetos de derecho colectivo. En este sentido, son dignos de destacar: la consagración de su preexistencia étnica y cultural a la presencia estatal en el territorio (derecho introducido con la reforma constitucional de 1994), y su denominación como "pueblos" y la definición de la totalidad del hábitat que ocupan y/o utilizan como "territorio" (ambos, conceptos aportados por el Convenio N 169 sobre Pueblos Indígenas y Tribales en Países Independientes de la Organización Internacional del Trabajo).

Por su parte, con la asunción del gobierno democrático en 1983, y de la mano de la creciente visibilidad de los pueblos indígenas en el escenario político provincial, 
Formosa también inició - de manera temprana en relación con los restantes Estados provinciales y aun con el nacional- la producción legislativa que delimita al sujeto indígena como objeto especial de la política de Estado. Las expresiones en ese sentido son dos. La primera, la sanción de la Ley Integral del Aborigen $N^{\circ} 426$ en 1984, que construye a un sujeto que, culturalmente distinto, apegado a tradiciones y costumbres propias, con un acceso deficiente a los servicios de educación, salud, vivienda y seguridad social, en otras palabras, en los márgenes de la ciudadanía, requiere de un Estado en un rol de protección, tutelaje y promoción en un grado mayor al demandado por los restantes ciudadanos (Briones et al., 2000). La segunda, la reforma de la Constitución provincial en 2003, por la que se reconoce la preexistencia de los pueblos indígenas que habitan la provincia. Decíamos que se trata de la construcción de un tipo particular de ciudadanía para el sujeto indígena.

\section{La comunidad Potae Napocna Navogoh: algunos antecedentes del conflicto}

En este marco, las acciones de reivindicación y demanda protagonizadas por los qom de Potae Napocna Navogoh en los últimos diez años se han fundado en un repliegue de la acción política sobre la comunidad y sobre organizaciones que agrupan a diferentes pueblos indígenas, en tanto nivel de lo colectivo. ${ }^{10}$ Con anterioridad, a mediados de la década de 2000, las formas de su acción política habían revelado su implicación en un proceso de lucha desplegado por pequeños productores de mercancías agrarias y trabajadores rurales criollos nucleados en el Movimiento Campesino de Formosa (MOCAFOR). Pero hacia 2007 el MOCAFOR experimentaba fracturas internas y un retraimiento de su presencia pública. Entonces, en un contexto jurídico en que el reconocimiento de derechos especiales sobre el que se funda la entrega de tierras para los pueblos indígenas en Formosa establece como forma de organización a la comunidad, los reclamos en torno a los que se movilizaban los qom anclaron cada vez más en su condición étnica particular, dejando de tener por eje la condición general dada por su experiencia como trabajadores. En 2011, tras realizarse una asamblea para la elección de sus representantes y en ocasión de inscribirse su personería jurídica ante el Registro Nacional de Comunidades Indígenas, la comunidad decidía: llevar el nombre de Potae Napocna Navogoh (Garra de Oso Hormiguero La Primavera), tal era nombrada por "los antiguos"; designar a la autoridad máxima como qarashe (líder junto a su pueblo); elegir un qaratagala (segundo

\footnotetext{
10 Nos referimos a: QOPIWINI, creada en 2015 con el objeto de nuclear a comunidades de los pueblos qom, pilagá, wichí y nivaclé; el Consejo de Comunidades Originarias, surgido en 2009 en procura de mejorar la calidad de vida de comunidades qom, wichí y pilagá de la provincia; el Consejo Plurinacional Indígena de Argentina, nacido con anterioridad al Bicentenario y que en 2010 marchó hasta Plaza de Mayo en la ciudad de Buenos Aires. Hoy en día, estas organizaciones ya no tienen relevancia-cuando no, existencia-(Cardin, comunicación personal).
}

líder) como otra autoridad; conformar seis consejos (de ancianas, ancianos, jóvenas, jóvenes, mujeres y varones); en breve, establecer "normas y pautas propias de nuestra organización comunitaria". ${ }^{11}$

Fue como parte de este proceso de movilización política protagonizado por los qom que, a fines de mayo de 2007, se llevó a cabo, en las deterioradas instalaciones de la antigua administración de la colonia, una reunión con funcionarios de la APN, del PNRP y de la DPOyRN de la SAyDS. ${ }^{12}$ También estaban presentes el director por el pueblo pilagá y el secretario del Instituto de Comunidades Aborígenes (ICA) provincial. El encuentro había sido convenido tras la denuncia del conflicto con el PNRP hecha tiempo antes por quien luego sería designado qarashe de la comunidad y electo como su representante ante las autoridades de la APN y de la DPOyRN de la SAyDS en la ciudad de Buenos Aires. El objetivo primordial era avanzar en la resolución del conflicto territorial entre la comunidad y el área natural protegida, cuya trayectoria ancla fuertemente en las maneras en que los qom fueron objeto de los proyectos (económicos, políticos, ideológicos) de exclusión /inclusión en el cuerpo de la nación (Lenton, 2014).

Dijimos que, en los inicios del curso histórico concreto que siguió el proceso de acumulación de capital en la región, el desarrollo de las distintas agroindustrias tuvo por condición de posibilidad la disponibilidad de mano de obra indígena. También dijimos cómo, en la introducción en determinadas prácticas laborales -que implicaba la colonización de la subjetividad de los indígenas en sus atributos materiales y mentales-, jugaron un papel insoslayable las reducciones y colonias estatales y las misiones religiosas. Sobre esta base, en 1940, por Decreto $N^{\circ} 80.513$, el gobierno nacional reservó una superficie de 5.000 ha de tierras correspondientes a las leguas a y b de la sección III de la Colonia Laguna Blanca, con destino a ser ocupada por la gente del cacique Trifón Sanabria. Por cierto, a partir de la llegada en 1937 de una misión de la iglesia protestante británica Emmanuel a cargo del pastor John Church al paraje Naineck (a 4 km al sudeste de la actual Potae Napocna Navogoh), se había concentrado gente indígena de sus alrededores (bandas de la parcialidad étnica conocida como takshek qom o "gente del este"). En 1952, por Decreto $N^{\circ}$ 3.297, las tierras reservadas para la gente de Sanabria

\footnotetext{
11 Véase la entrada titulada "Un momento histórico para nuestra comunidad", del 30 de julio de 2011, en: http://comunidadlaprimavera. blogspot.com/search?updated-max $=2011-09-15 T 12 \% 3 A 31 \% 3 A 00$ 03\%3A00\&max-results $=110$

12 Según la Resolución N 58/2007 de la Jefatura de Gabinete de Ministros, eran propósitos de la DPOyRN: planificar acciones en materia de política ambiental con relación a los pueblos indígenas; promover su participación activa en las acciones de la Secretaría; desarrollar programas de capacitación dirigidos a dichos pueblos para la gestión de sus recursos naturales; asistirlos en esa gestión, teniendo en cuenta su derecho a fijar las prioridades del desarrollo desde su cosmovisión; coadyuvar en la implementación de proyectos de desarrollo local de los pueblos indígenas.
} 
se constituyeron en colonia bajo la jurisdicción de la Dirección de Protección al Aborigen -creada en el ámbito de la Secretaría de Trabajo y Previsión de la Nación y encargada de la adquisición de implementos agrícolas y ganado con destino a las colonias existentes-.

Luego de la provincialización de Formosa en 1955, la colonización con indígenas fue contemplada en el marco de una ley de carácter general. La Ley $N^{\circ}$ 113/60 de Colonización y Tierras Fiscales señalaba que el ciudadano indígena podía ser adjudicatario de tierra fiscal, previa acreditación de su capacidad para ello, y preveía planes especiales de habilitación con objeto de conseguir el aprovechamiento integral, racional e intensivo de la tierra, invirtiéndose su producto en la adquisición de máquinas y herramientas para uso de los indígenas. Por Decreto $\mathrm{N}^{\circ} 1.363 / 63$, los qom obtuvieron la aprobación de una mensura de 5.107 ha. La escrituración fue realizada en 1985, tras la sanción de la Ley N 426/84, bajo la forma de un título comunitario a nombre de la Asociación Civil Comunidad Aborigen La Primavera, sobre una superficie de 5.187 ha (De la Cruz, 2005).

Esta extensión de tierras comprende el sector denominado "el triángulo": tierras con que la provincia buscó compensar a la comunidad tras permitir la continuidad de la ocupación de la familia criolla Celía en tierras que fueran reservadas por el decreto de 1940 a la comunidad (Cardin, 2013a). ${ }^{13}$ Tanto "el triángulo" como una porción de la superficie de la laguna Blanca (también incluida en las tierras reservadas en 1940), están, a la vez, bajo jurisdicción del PNRP. En otras palabras, aun a pesar de la reducción de su extensión, el PNRP comprende tierras reservadas para los qom en 1940 y otorgadas a la comunidad en 1985; lo que es más importante aún, esta superposición de mensuras encuentra en su base la desposesión del territorio de ocupación tradicional de los qom ${ }^{14}$ (Figura 1).

\section{La relación entre los qom de Potae Napocna Navogoh y la naturaleza: prácticas actuadas y sentidos atribuidos}

La superposición de mensuras entre las tierras incluidas en el título de propiedad otorgado a la comunidad y aquéllas comprendidas en el del PNRP devenía demanda durante la reunión celebrada con funcionarios del PNRP, de la APN y de la DPOyRN: en principio, la necesidad de precisar los límites entre unas y otras. Mientras que el intendente del PNRP sostenía que "para la ley de Parques, para la

\footnotetext{
13 En 2007, alrededor de 700 ha de las tierras ocupadas por la familia Celia fueron vendidas a la provincia para la instalación de una sede de la Universidad Nacional de Formosa. Esto detonaría futuras acciones de reivindicación y demanda.

14 Para un análisis de la delimitación de dicho territorio en el marco del proceso de relevamiento territorial realizado a propósito de la Ley $N^{\circ} 26.160$ de Emergencia en materia de posesión y propiedad de las tierras que tradicionalmente ocupan las comunidades indígenas originarias del país, véase Cardin (2018).
}

mensura de Parques, la laguna está dentro del parque" (Ricardo, 30/05/2007), ${ }^{15}$ varios qom tomaban la palabra para expresar de manera coincidente que:

“Ese es el tema. La laguna está a nombre del Parque, pero hay una parte que nos corresponde a nosotros. Y los guardaparques pasan los límites de la laguna hacia donde estamos. Salen de la laguna y recorren la costa de nuestra casa. Nosotros queremos libertad de pescar y acceder al agua. Eso es lo que queremos. Definamos la mensura. Y definamos el manejo de los recursos" (Damián, 30/05/2007).

Claro que aquella superposición de mensuras se traducía en situaciones de conflicto, y más aún de abuso, de carácter local y cotidiano. De ellas daban cuenta distintos mariscadores de la comunidad: ${ }^{16}$

“Era un día jueves a la mañana. Nosotros entramos en el agua. Sacamos una carnada para mantener nuestros chicos, para mantener nuestra familia. Yo, por lo menos, soy hombre casado, de 55 años, no tengo nada de beneficios. No es que me gusta entrar en el agua, pero por una necesidad, para mantener a los chicos. Al otro día, a las 8 , vinieron los parqueros, nos sacaron toda nuestra red y nuestros baldes y todo lo que sacamos, la carnada. Echaron a perder otra vez. Y un sacrificio para nosotros. Quemaron toda nuestra ropa, el mismo día, llevaron nuestra frazada. Todo lo que tenemos. Y venimos así con shorcito, en el monte. Pero sabemos que es terreno nuestro, por eso no tengo vergüenza, porque es nuestro. $Y$ todo lo que nosotros sacamos es carnada, nosotros vendemos, para mantener a nuestros chicos. Eso nomás. Perdemos todo" (Juan, 30/05/2007).

Por cierto, en ocasión de la reunión, la demanda giró sobre el acceso a la laguna y los recursos en ella contenidos. Sus prácticas de producción desplegadas en torno a ellos son presentadas, en el Plan Quinquenal de Manejo 2007-2011 elaborado para el PNRP, como un problema -al que se hacía referencia, en los términos de una "inadecuada" utilización de los recursos naturales (Administración de Parques Nacionales, 2006: 13)-. En un contexto de progresiva mutilación de los atributos productivos de la fuerza de trabajo indígena, ${ }^{17}$ el reclamo

\footnotetext{
${ }^{15}$ Si bien la reunión fue definida como "pública" y su registro como una forma de documentar "lo que está pasando", los nombres de las personas citadas en el trabajo son seudónimos.

16 Por marisca se entiende la caza de animales silvestres, la pesca, la recolección de frutos silvestres y miel.

17 Esta mutilación encontraba múltiples expresiones: la participación decreciente en el cultivo del algodón, como productores independientes y asalariados estacionales, ante la concentración y centralización del capital en la producción algodonera y ante la caída en la escala de esa producción y el avance de la mecanización de la cosecha; el arrendamiento de sus parcelas de tierra a terceros por
} 


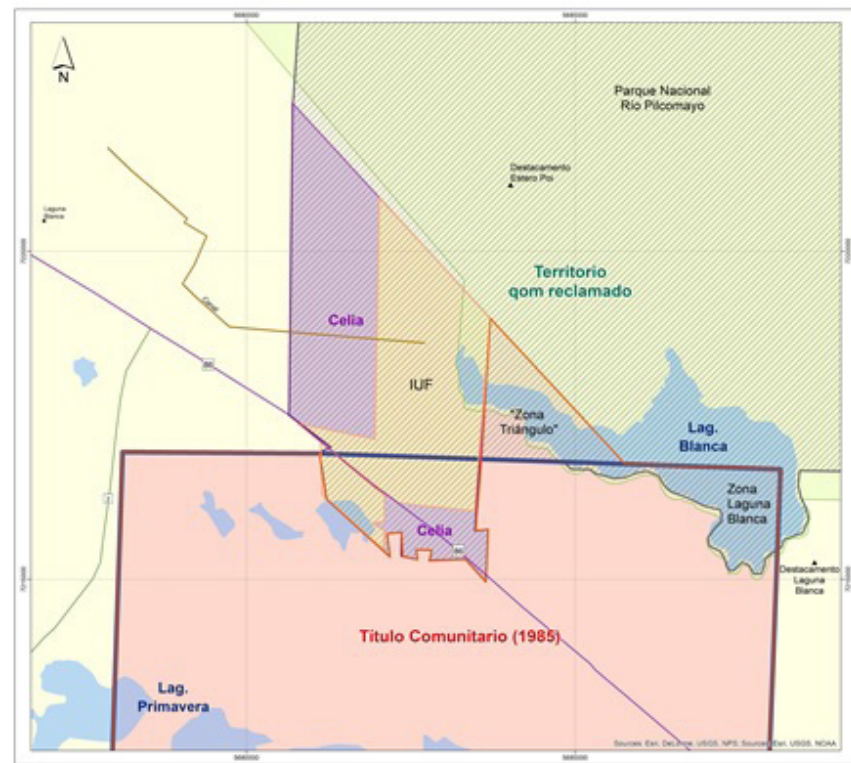

de los qom era por la posibilidad de la continuidad de una práctica (la marisca) que, sin ser central, es una a la que pueden recurrir a la hora de la (re)producción social de su vida. Se trata de una práctica que, entre los qom, aparece teniendo a la necesidad y al sacrificio como formas necesarias. En todo caso, era la dimensión material de la relación con el territorio la que aparecía en primer plano en la formulación de la reivindicación. ${ }^{18}$ En este sentido, reclamaban ante el requisito de portar un permiso para practicar la pesca en la laguna. Esta exigencia tenía lugar en el marco de una normativa que prohíbe la pesca dentro de la jurisdicción del parque sobre la base de su concepción, junto con la caza, como una "presión sobre los recursos naturales" (Entrocassi Fassinato y Espínola, 2006: 11). En efecto, en el mencionado plan quinquenal, se identificaban como obstáculos para el cumplimiento de los objetivos de conservación del patrimonio natural del PNRP la pesca y la caza furtivas. Resultaba particularmente problemática, en términos de su impacto negativo, la "caza de recreo y status" en tanto se trataba de aquélla mayormente extendida; por su parte, la "caza de subsistencia y cultural" atribuida a los qom era vista como insignificante y ocasional (Administración de Parques Nacionales, 2006: 46). Si bien la pesca también es considerada una actividad esporádica y de baja escala, resulta central en el reclamo de los qom; éste asumía los siguientes términos:

la falta de herramientas e insumos para ponerla en producción, y su posterior asalarización como carpidores y cosecheros en sus propias tierras; la caída del salario obtenido, que apenas alcanzaba a cubrir la reproducción física durante el período de trabajo; la venta de los productos del trabajo artesanal y de los subproductos de la marisca por debajo del precio al que luego circulaban en el mercado; la constitución generalizada en beneficiarios de programas sociales de asistencia al desempleo y a la pobreza (J. Iñigo Carrera y V. Iñigo Carrera, 2017).

${ }^{18}$ Por cierto, la producción social del espacio en tanto territorio -o, lo que es lo mismo, el proceso de territorialización- comprende tanto el dominio (político-económico) como la apropiación (simbólicocultural) de ese espacio por los colectivos humanos, en el marco de un complejo y variado ejercicio de poder (Haesbaert, 2011).

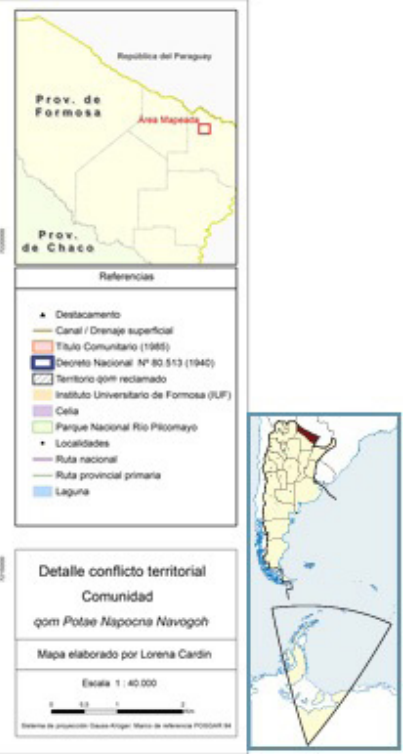

Figura 1. Tierras y territorio en conflicto (comunidad Potae Napocna Navogoh). Fuente: Elaborado por Lorena Cardin en el marco de la demanda judicial ante la Corte Suprema de Justicia de la Nación.

Figure 1. Lands and territory in conflict (Potae Napocna Navogoh community). Source: Elaborated by Lorena Cardin for presentation in the context of the lawsuit before the Supreme Court of Justice of the Nation.

"Nosotros hemos planteado sobre este tema al intendente [del Parque] [...] sobre, o sea, para pescar. Y lo que nos pedía era que nosotros, el intendente tiene que ir a la casa del indígena para verificar si es pobre, si no tiene plan [de asistencia a la pobreza y el desempleo], o no tiene pensión, si no tiene acceso a las mercaderías. Entonces si te dan permiso en Laguna Blanca, en la oficina del intendente te da una nota, esa nota tenés que entregar al responsable del destacamento del Parque, y ése te tiene que acompañar hasta la laguna con el bote. Y solamente está permitido sacar dos pescados. Pero tenés que hacer estos trámites. Mientras la familia se está muriendo de hambre. ¿Por qué el no puede entender esa cosa? Porque es una situación indígena, de la cual la mayoría de los hermanos que entran a la laguna no tienen beneficios" (Juan, 30/05/2007).

En un contexto de falta de red de abastecimiento de agua potable y de consumo, en el mejor de los casos, de la extraída de los pozos de las escuelas de la comunidad, hasta el agua misma de la laguna era objeto de la prohibición: "Un día me fui, nosotros tomamos el agua de la laguna. Con toda mi familia sacamos para tomar. $Y$ un día encontramos con el parque, y ellos casi corrieron a mi señora, hijo, hija. Tengo siete familias. Ellos vinieron corriendo a mi señora, mi hija. Todas las personas que entran ahí, ellos corren, sacan. Tenemos restringido el acceso al agua" (Rubén, 30/05/2007).

En las condiciones de existencia del presente se evidencia, por un lado, el despojo del territorio y de los recursos en él comprendidos, y por otro, la ya mencionada mutilación de los atributos productivos en su condición de trabajadores; tanto uno como otra, producto del avance del capital. En su alocución, un miembro de la comunidad que había vivido varias décadas en un barrio periférico a la ciudad de Formosa y tiempo antes nos había expresado su deseo 
de volver al "campo" para "poder trabajar, poder otra vez vivir la naturaleza, lo que la naturaleza me ofrece" (Pedro, 11/09/2004), decía:

"Están arruinando el suelo de la comunidad [con la utilización de semillas transgénicas y agroquímicos]. Ese es un daño. [La integrante del Directorio de la APN pregunta por quién alquila y utiliza agroquímicos]. Un empresario de apellido Pagliari, miles de hectáreas tiene. [...]. Yo hace tres años que estoy acá. No sabemos defendernos, porque yo no soy abogado [...]. Los propios indígenas manejan el agua, cuando no había pensión, no había empleado, no había beneficio para nada. No hay depredación animal, tampoco. [...] Cuando el gobierno piensa vender las tierras, y los indígenas «es nuestro, acá vamos a tener pescado», pero cuando llegan los dueños van a meter topadoras en las tierras y van a plantar soja transgénica. [...]. «No, si el gobierno nos va a dar beneficio del gobierno», [...] vamos a ser beneficiados con nuestra entrada, va a haber una vivienda, va a haber escuela, va a haber aljibe, va a haber comedor, va a haber pensión, va a haber cajas de alimentos [el tono va in crescendo]. El beneficio [...] con todos los instrumentos para que el hombre pueda trabajar [...]. Si el gobierno tiene buena intención, el gobierno te va a dar. [...]. Empiezan a envenenar nuestro lugar. Nos están matando de diferentes formas. Prohibido para que nadie vaya a pescar, que nadie vaya a sacar una miel, que nadie vaya a matar un ñandú, que nadie saque un pescado. [...] relación libre con la naturaleza [...] doqshé [blanco] maneja, nosotros no manejamos, y ellos hacen todas las leyes en beneficio [propio]" (Pedro, 30/05/2007).

Anclada en un pasado de abundancia, control y no dominación -que en su tensión, no carente de ambigüedades, con un presente de pérdidas (Gordillo, 2006) no hace sino evidenciar la trayectoria histórica seguida por los qom en tanto sujetos productivos-, la relación con la naturaleza propia de los qom, aquella para cuyo desarrollo hoy encuentran obstáculos, se representa libre. Aun cuando no desconoce la transformación que realizan del medio físico con el objeto de producir sus medios de vida, ésta asume un carácter armónico. Se constituye, a la vez, en una de las expresiones más paradigmáticas de la producción de un "ser-esencialcompartido", de la producción de una etnicidad particular en tanto inmanente a la condición qom como manifestación de su ser, irreductible y primario, biológico o cultural (Restrepo, 2004: 29). Se trata, en breve, de una manera de entender la etnicidad que, en su adopción de un cariz esencialista, se revela propia de los sujetos indígenas. Un día después de la reunión analizada, un líder político de la comunidad refería:
"La naturaleza es parte de nuestra existencia. Somos de la naturaleza. Como en aquellos tiempos [...] entonces eran elementos naturales que nos servían mucho a nosotros, el bosque, el agua, la tierra, es una cosa que da vida a nosotros. Y en este tiempo que nosotros hemos podido mantener esa forma de mantener la naturaleza, porque queremos conservar en agradecimiento de nuestra existencia, el aporte para nuestra vida. [...] así que nosotros tenemos deuda con la naturaleza, porque nos dio vida, a través de la existencia de nuestros antepasados hasta esta altura del tiempo que hoy vivimos, porque la naturaleza nos dio los frutos, el calor, los animales, cosas que no se compran. [...]. Forma parte de la propia cultura, formamos parte de la misma naturaleza. Por eso el indígena siempre lucha por un monte, cuida el río, los pájaros, tantas cosas cuidamos, porque son nuestros hermanos" (Antonio, 31/05/2007).

La relación de carácter natural que el indígena sostendría con su medio físico devendría garantía de su adecuada conservación, una propia de su "forma de pensar". Y, en su constitución en característica esencial como pueblo, deviene también sustento de las formas de acción política desplegadas en el marco de las luchas indígenas, en particular, por el territorio. ${ }^{19}$

Pero la apologética de la relación directa y armónica con la naturaleza en tanto atributo natural de la condición étnica es propia también de la política de Estado que tiene por objeto a los pueblos indígenas, siendo corriente entre quienes personifican su estructura. En tanto no cabía duda del carácter de pueblo indígena de los qom de Potae Napocna Navogoh, así se manifestaba quien se desempeñaba en el Directorio de la APN:

"muchos años atrás, fue pensado el manejo de estos Parques Nacionales como un lugar cerrado que debía conservarse sin que nadie lo tocara y protegido de la gente. Ese concepto, esa forma de

\footnotetext{
19 Más allá de su esencialización como un componente de la acción política, aquella relación encuentra su sustento positivo e histórico en la erección de la tierra en condición material de producción. Se sabe que históricamente, y en tanto era limitado el desarrollo de las fuerzas productivas del trabajo, la caza, la pesca y la recolección implicaban poco cambio de forma de los objetos apropiados: los frutos del suelo eran consumidos como medios de vida más o menos directamente. Se trata de prácticas comúnmente asociadas a una forma no productiva de transformación de la naturaleza. Pero, lejos de ser abstractamente improductivas, las formas de trabajo implicadas en la marisca resultan productivas desde el punto de vista material de la producción social -en tanto producen valores de uso-, desde el punto de vista de la organización de la producción social a través del mercado -en tanto producen valor-y desde el punto de vista de la organización capitalista de la producción social -cuando de lo que se trata es de la producción de plusvalía- (V. Iñigo Carrera, 2013). Si bien la marisca no constituye la expresión más evidente de la falsedad de la ajenidad de las prácticas productivas de los qom a la organización capitalista de la producción social, mencionamos cómo los indígenas chaqueños se constituyeron tempranamente en brazos baratos para la expansión del capitalismo regional.
} 
pensar, ha ido cambiando con el tiempo. Hoy en día, esta gestión de Parques Nacionales, entiende, nosotros pensamos que no puede conservarse un área de la naturaleza sin que lo hagamos juntos, los que nos ocupamos específicamente del tema y nuestros vecinos, quienes están. Necesitamos de nuestros socios, nuestros amigos para proteger esa naturaleza. Porque hoy en día nuestro territorio tiene fuertes peligros. Lo que mencionaba hace un rato [Pedro], peligros que corresponden a intereses económicos. Y que nos afecta a la forma de vida, la salud, la integridad misma del territorio. Entonces Parques a lo largo de todo el país es una función importante no sólo para nosotros sino para los que viven acá, para los chicos. [...]. Entonces nosotros como Parques Nacionales no renegamos de la función que tenemos como defensores de la naturaleza, pero estamos convencidos que también necesitamos ayuda. En este sentido, las comunidades indígenas son amigos que tienen en su forma de pensar muchos valores parecidos, de respeto a la naturaleza, poner en valor a la naturaleza. Entonces pensamos que las comunidades pueden, quisiéramos que fueran nuestros acompañantes en este trabajo. [...]. Ahora, por otra parte, si bien esa idea original, del parque cerrado y que no podía entrar nadie, ha ido cambiando, todavía en partes existe un poco de todo, gente de la vieja escuela, gente que tiene una forma de pensar más abierta. Ahora lo que yo sí puedo comprometer, en nombre del directorio y del mío personal, que está orientada en esta línea, de tener una fuerte vinculación con las comunidades [...], que participen de las actividades dentro del parque, sobre todo las vinculadas a la cultura, la forma de la visión y la cultura. Creemos que la naturaleza la tenemos que defender entre todos" (Cecilia, 30/05/2007).

Si la conservación y el futuro de los niños eran los propósitos, la comunidad era convocada a perseguirlos -en el marco del nuevo paradigma en la relación entre las áreas protegidas y los pueblos indígenas- sobre la base de una condición en la que la relación con la naturaleza se constituía en uno de los atributos naturales de sus miembros: naturalmente cercanos a y protectores de la naturaleza. Por su parte, los derechos derivados de esa misma condición (indígena), reconocidos nacional e internacionalmente, que tenían por objeto al territorio y al manejo de los recursos en él contenidos, parecían no encontrar un lugar en las palabras de la integrante del Directorio de la APN. Tras señalar la extemporaneidad del carácter de la relación entre los qom y el PNRP, así lo hacía notar quien entonces presidía la DPOyRN:

"Yo digo una cosa, y lo digo con todo el respeto. [Cecilia] dice «acá hay una ley de Parques» [referida a la prohibición de pescar en su jurisdicción]. Pero nosotros somos un pueblo preexistente, existimos antes que existiera Parques. Tenemos leyes hoy, leyes constitucionales. Y una ley de Parques no puede ser más fuerte que una Constitución que reconoce el derecho nuestro como pueblo indígena de gestionar nuestros recursos" (Roberto, 30/05/2017).

Crítico de la manera en que la integrante del Directorio de la APN se refería a la relación entre su institución de pertenencia y los pueblos indígenas, planteaba el vínculo de estos últimos con la naturaleza en idénticos términos:

\begin{abstract}
"A mí no me gusta tanto la palabra amigo. Nosotros no somos amigos, no queremos actuar como amigos de Parques para cuidar la naturaleza. Nosotros existimos, somos partes de la naturaleza, somos preexistentes a Parques, existimos desde hace miles de años. La forma de relacionarnos con el medio ambiente ha sido desde nuestra cosmovisión, nuestra cultura. O sea, si alguien que puede [...] de cómo conservar la naturaleza son los pueblos originarios. Y eso está reconocido en el mundo. Parques también lo ha reconocido por eso tenemos una relación de comanejo en el sur y en otros parques" (Roberto, 30/05/2007).
\end{abstract}

Dos son los argumentos sobre los que se sostiene la construcción de los pueblos indígenas como guardianes o protectores de la naturaleza: que la caza, la pesca y la recolección que practican no van más allá de sus necesidades alimentarias y que la cosmovisión indígena no concibe la acumulación ni el propósito de lucro. Parece ser ésta una construcción común. Ahora bien, se trata de una construcción que no hace sino esconder la fijación del indígena a la naturaleza y a "pautas culturales ancestrales", ignorando sus efectivas condiciones materiales de existencia. Por cierto, en un contexto en que la naturaleza es cada vez más objeto de una extrema apropiación privada para la puesta en producción capitalista, la posibilidad de la continuidad de la práctica de la marisca se encuentra fuertemente limitada, y la preservación de aquellas pautas esconde la perpetuación de los indígenas en condiciones miserables de vida.

\section{Consideraciones finales}

En la reunión del mes de mayo de 2007, fue la dimensión material de la relación con el territorio ocupado la que apareció en primer plano en la formulación de la reivindicación. Cuatro años más tarde, los qom de Potae Napocna Navogoh habían alcanzado notoriedad a nivel nacional tras haber sostenido un prolongado corte de la ruta $N^{\circ} 86$ en reclamo del fin del avance sobre su territorio por parte de capitales privados y los Estados y haber irrumpido el conflicto en la ciudad de Buenos 
Aires mediante un acampe sostenido por cerca de seis meses, luego de que la policía provincial pusiera fin de manera violenta a dicho corte. Entonces, la superposición de mensuras entre el parque y las tierras reclamadas por la comunidad seguía formando parte de la agenda de demandas de los qom -aún hoy dicha superposición no ha encontrado una resolución-. A mediados de mayo de 2011, su qarashe dirigía las siguientes palabras al Consejo Directivo de la Facultad de Filosofía y Letras de la Universidad de Buenos Aires:

“Para nosotros es interesante el apoyo que nos brindan para seguir avanzando en el tema de la recuperación del territorio, que nos es fundamental porque está ligado a nuestra existencia. Se trata de un territorio que mantuvimos siempre a través del uso y de la relación con la naturaleza. Lo más importante de todo es que hay una laguna que para nosotros es fundamental, porque es un sitio sagrado. ${ }^{20}$ Aparte de la relación espiritual, hay seres sobrenaturales que habitan esa laguna. Para nosotros es mucho más que los recursos económicos. [...]. Desde hace miles de años hemos podido proteger y cuidar ese espacio, que nos es fundamental porque ahí residen nuestra alimentación, nuestra fortaleza espiritual [...]. La única manera para superar los momentos difíciles es justamente la relación con este espacio físico, que nos fue despojado por Parques Nacionales. [...]. Ya no podemos ingresar a buscar los recursos naturales para poder subsistir. [...]. Por eso la importancia de poder recuperar ese monte, porque es un medio de vida. [...]. Queremos estar en nuestro territorio para poder desarrollar nuestra cultura. Nosotros entendemos la tierra como la vida: no se negocia ni se vende. Por eso es importante que nos devuelvan la tierra, es como volver a revivir, para poder hacer algo a favor de esta cultura que ha sido silenciada por miles de años". ${ }^{21}$

Aquí, se alude a la especial importancia que para este pueblo reviste su vínculo con el territorio, comprendiendo no sólo su dimensión material sino también la simbólica. En este último sentido, se deja entrever, por otra parte, "una continuidad culturalmente-establecida -opuesta a una separación- entre los mundos naturales, humanos y sobrenaturales" (Escobar, 2003: 78). Se trata, aquí, de la (re)presentación pública de la etnicidad en términos de ciertas características biológicas y/o culturales asumidas

\footnotetext{
20 El carácter sagrado atribuido a la laguna fue invocado asimismo durante la audiencia pública convocada por la Corte Suprema de Justicia de la Nación en 2012 con el objeto de que las distintas partes involucradas en la causa iniciada por los qom contra la provincia de Formosa en reclamo de tierras dieran a conocer su parecer sobre el conflicto (Cardin, 2013b).

21 Palabras incluidas en los considerandos de la Declaración del mencionado Consejo Directivo del 31 de mayo de 2011, en apoyo a los reclamos de la comunidad Potae Napocna Navogoh.
}

como rasgos evidentes y naturales de esa condición; (re)presentación que legitima su específica intervención política con vistas a demandar derechos derivados de esa condición étnica (Restrepo, 2004). Una aproximación dominante en la literatura que analiza luchas indígenas por derechos, recursos, sentidos es la que concibe a la identidad como una construida en términos esencialistas de manera estratégica como parte de acciones de reivindicación y demanda (Conklin, 1997; Hale, 1996).

Más allá de la crítica a los riesgos políticos en ella encerrados (acusaciones de oportunismo, inautenticidad), podríamos decir que en aquella particular construcción -dada en determinadas coyunturas- de una identidad indígena reconocible a partir de la selección y combinación de ciertos elementos está involucrada la agencia de los qom (su posicionamiento, su articulación) (Li, 2000). Sin dejar de problematizar la concepción de la etnicidad desde el esencialismo, resulta necesario repensar esa construcción en términos de una estrategia o de una contingencia como parte de la dinámica propia de las formas de acción política indígena en tanto configuradoras de sujetos políticos colectivos. En un trabajo anterior (V. Iñigo Carrera, 2008), realizamos una crítica al uso de la noción de estrategia a la hora de hablar de la relación social de los qom. Decíamos que lejos estábamos de entender las diversas formas que esta última asume en los términos de una multiplicidad de estrategias de reproducción adoptadas por actores pensados como individuos abstractamente libres con el objeto de obtener los medios de vida necesarios para producir su vida. Estábamos lejos, decíamos, de pensar en múltiples posibilidades para la producción de su vida entre las que los qom sólo deberían elegir, tan pronto como la voluntad individual sólo es portadora de determinaciones más generales que la trascienden (Marx, 2001). Estas consideraciones entonces vertidas nos resultan sugestivas, e igualmente válidas, al momento de desarrollar analíticamente las formas de acción política indígena.

No obstante atravesada por un montaje de naturalizaciones -que se expresa tanto en la multiplicación de formas de acción política indígena que encuentran uno de sus ejes en la lucha por el territorio y los recursos naturales en él comprendidos como en el desarrollo de un nuevo paradigma en conservación-, la subjetividad indígena que se construye es una que no es sino producto de su determinación como atributo de la relación social objetivada propia del modo de producción capitalista. En tanto tal, es la privación a esta porción de la población trabajadora del ejercicio de su capacidad para participar activamente en el proceso de producción y consumo social la relación económica que se realiza bajo la forma de la producción de una subjetividad indígena esencialmente diferencial y con la convergencia entre acumulación de capital y conservación como telón de fondo.

San Carlos de Bariloche, 2 de abril de 2019 


\section{Agradecimientos}

Este trabajo es producto de la labor de investigación desarrollada entre 2004 y 2014 en el marco de sucesivos proyectos financiados por el Consejo Nacional de Investigaciones Científicas y Técnicas y la Universidad de Buenos Aires. Agradecemos a los/as evaluadores/as anónimos/as cuyos comentarios enriquecieron su versión final. También, a Guillermo Quiña y Lorena Cardin por su lectura y revisión crítica de versiones previas; Lorena cedió, asimismo, el mapa incluido en el trabajo. Por su parte, Laura Starópoli, del Programa Pobladores y Comunidades de la Dirección Nacional de Conservación de Áreas Protegidas, y Catalina Coali, de la Biblioteca Central y Centro de Documentación Perito Francisco P. Moreno, ambas dependencias de la Administración de Parques Nacionales, facilitaron y colaboraron con la consulta de materiales relacionados con el Parque Nacional Río Pilcomayo.

\section{Bibliografía}

Administración de Parques Nacionales (1999). Plan Operativo Anual 2000. Parque Nacional Río Pilcomayo, Formosa - Argentina. Laguna Blanca: Autor.

Administración de Parques Nacionales (2006). Plan Quinquenal de Manejo 2007-2011. Parque Nacional Río Pilcomayo, Formosa - Argentina. Laguna Blanca: Autor.

Aylwin, J. (2011). Conservación en territorios indígenas: marcos jurídicos y experiencias nacionales y comparadas y directrices internacionales. En J. Aylwin y X. Cuadra (Eds.), Los desafíos de la conservación en los territorios indígenas en Chile (pp. 9-91). Temuco: Observatorio Ciudadano.

Bello, A. (2009). Multiculturalismo, ciudadanía y pueblos indígenas. ¿Un debate pendiente en América Latina? En L. Valladares de la Cruz, M. L. Pérez Ruiz y M. Zárate (Coords.), Estados Plurales: Los retos de la diversidad y la diferencia. México DF: Editorial UAM - Iztapalapa Juan Pablos Editor.

Briones, C., Carrasco, M., Escolar, D. y Lenton, D. (septiembre, 2000). El espíritu de la ley y la construcción jurídica del sujeto "pueblos indígenas". VI Congreso Argentino de Antropología Social. Universidad Nacional de Mar del Plata, Mar del Plata, Argentina.

Brockington, D. y Duffy, R. (2010). Capitalism and Conservation: the Production and Reproduction of Biodiversity Conservation. Antipode, 42(3), 469-84.

Brodersohn, V. y Slutzky, D. (1975). Diagnóstico de la estructura social de la región NEA. Formación y desarrollo de las estructuras agrarias regionales: Misiones y Formosa. Buenos Aires: Consejo Federal de Inversiones.
Cardin, L. (julio, 2013a). La comunidad qom Potae Napocna Navogoh (La Primavera) y el proceso de lucha por la restitución de su territorio. $X$ Jornadas de Sociología. Universidad de Buenos Aires, Buenos Aires, Argentina.

Cardin, L. (2013b). Construcciones en disputa de la identidad qom. La escenificación de las diferencias ante la Corte Suprema de Justicia de la Nación. En F. Tola, C. Medrano y L. Cardin. (Eds.), Gran Chaco. Ontologías, poder, afectividad. (pp. 361-384). Buenos Aires: Rumbo Sur.

Cardin, L. (2018). Entre realidades y simulacros. El proceso de relevamiento del territorio qom. M. Carrasco (Comp.), Campos de interlocución y políticas de reconocimiento indígena en Argentina (pp. 19-36). Buenos Aires: Antropofagia.

Carenzo, S. y Trentini, F. (2014). El doble filo del esencialismo "verde": repensando los vínculos entre pueblos indígenas y conservación. En H. H. Trinchero, L. Campos Muñoz y S. Valverde (Eds.), Pueblos indígenas, estados nacionales y fronteras. Tensiones y paradojas de los procesos de transición contemporáneos en América Latina (pp. 103-134). Buenos Aires: Facultad de Filosofía y Letras, Universidad de Buenos Aires - CLACSO.

Carpinetti, B. (2007). Una experiencia intercultural de comanejo entre el Estado y las comunidades mapuches en el Parque Nacional Lanin, Argentina. Santiago de Chile: Organización de las Naciones Unidas para la Agricultura y la Alimentación.

Conklin, B. (1997). Body Paints, Feathers, and VCRs: Aesthetics and Authenticity in Amazonian Activism. American Ethnologist, 24(4), 711-737.

Conklin, B. y Graham, L. (1995). The Shifting Middle Ground: Amazonian Indians and Eco-Politicis. American Anthropologist, 97(4), 695-710.

De la Cruz, L. M. (2005). Situación jurídica y territorial de las comunidades indígenas de la provincia de Formosa (Argentina). Manuscrito inédito.

Entrocassi Fassinato, M. y Espínola, R. (2006). Toponimia del Parque Nacional Río Pilcomayo. Buenos Aires: Administración de Parques Nacionales.

Escobar, A. (2003). "Mundos y conocimientos de otro modo". El programa de investigación de modernidad/ colonialidad latinoamericano. Tabula Rasa, 1, 51-86. Recuperado de https://www.redalyc.org/ html/396/39600104/index.html

Ferrero, B. (2014). Conservación y comunidades: una introducción. Avá, 24, 11-33. Recuperado de http://www. 
scielo.org.ar/scielo.php?script=sci_arttext\&pid=S185116942014000100001 \&lng=es

Ferrero, B., Gómez, C. y Franco, A. (noviembre-diciembre, 2015). Cuando el turismo manda. Guardaparques enfrentados a la alianza entre guaraníes y empresas forestales en el norte argentino. XI Reunión de Antropología del Mercosur. Universidad de la República, Montevideo, Uruguay. Recuperado de http://xiram.com. uy/actas-del-congreso/grupos-de-trabajo/ponenciasgrupo-de-trabajo-33

Gordillo, G. (2006). En el Gran Chaco. Antropologías e historias. Buenos Aires: Prometeo.

Guy, D. (2000). “El Rey Algodón”. Los Estados Unidos, la Argentina y el desarrollo de la industria algodonera argentina. Mundo Agrario, 1(1). Recuperado de https://www.mundoagrario.unlp.edu.ar/article/view/ MAv01n01a01/1554

Haesbaert, R. (2011). El mito de la desterritorialización. Del "fin de los territorios" a la multiterritorialidad. México: Siglo XXI.

Hale, C. (1996). Mestizaje, Hybridity and the Cultural Politics of Difference in Post-Revolutionary Central America. Journal of Latin American and Caribbean Anthropology, 2(1), 34-61.

Iñigo Carrera, J. (2012). Acerca del carácter de la relación base económica - superestructura política y jurídica: la oposición entre la representación lógica y la reproducción dialéctica. En G. Caligaris y A. Fitzsimons (Eds.), Relaciones económicas y políticas. Aportes para el estudio de su unidad con base en la obra de Karl Marx (pp. 8-19). Buenos Aires: Facultad de Ciencias Económicas, Universidad de Buenos Aires.

Iñigo Carrera, J. (2013). El capital: razón histórica, sujeto revolucionario y conciencia. Buenos Aires: Ediciones Cooperativas.

Iñigo Carrera, J. e Iñigo Carrera, V. (2017). Capitalismo y pueblos indígenas en el Chaco argentino: formas y determinaciones de una subjetividad productiva. Antropologías del Sur, 7, 117-139. Recuperado de http:// revistas.academia.cl/index.php/ads/article/view/788/913

Iñigo Carrera, V. (2008). Sujetos productivos, sujetos políticos, sujetos indígenas: las formas de su objetivación mercantil entre los tobas del este de Formosa. (Tesis de doctorado no publicada). Facultad de Filosofía y Letras, Universidad de Buenos Aires, Buenos Aires.

Iñigo Carrera, V. (2011). Configuraciones de la relación de ciudadanía entre los tobas de Formosa: lo universal y lo particular. Andes, 22, 219-246. Recuperado de http://www.scielo.org.ar/scielo.php?script=sci_
arttext\&pid=S1668-80902011000200002\&lng=es\&tln $\mathrm{g}=\mathrm{es}$

Iñigo Carrera, V. (2013). Trabajadores indígenas en el Chaco argentino: algunos sentidos estigmatizadores. Antípoda. Revista de Antropología y Arqueología, 17, 229-251. doi: http://dx.doi.org/10.7440/antipoda17.2013.13

Iñigo Carrera, V. (2014). Un cultivo para los qom: la política de Estado en la producción de una trayectoria como trabajadores y productores del algodón en el Chaco central. En M. Ruffini y A. Salomón (Comps.), Estado, políticas públicas y ciudadanía en el mundo rural (pp. 85-99). Buenos Aires: Imago Mundi.

Iñigo Carrera, V. (2015). Naturaleza y naturalización en las luchas políticas de los qom del este formoseño. Identidades, 8, 153-171. Recuperado de https:// iidentidadess.wordpress.com/numeros-anteriores/ numero-8/

Kelly, A. (2011). Conservation Practice as Primitive Accumulation. The Journal of Peasant Studies, 38(4), 683-701. doi: 10.1080/03066150.2011.607695

Lenton, D. (2014). De centauros a protegidos. La construcción del sujeto de la política indigenista argentina desde los debates parlamentarios (1880-1970) (Tesis de doctorado). Corpus. Archivos virtuales de la alteridad americana, 14(2). doi: 10.4000/corpusarchivos.1290

Li, T. (2000). Articulating Indigenous Identity in Indonesia: Resource Politics and the Tribal Slot. Comparative Studies in Society and History, 42(1), 149-179.

Marx, K. (2001). El Capital. Crítica de la Economía Política. México DF: Fondo de Cultura Económica.

Navarro Floria, P. (2007). La Comisión del Paralelo $41^{\circ}$ (1911-1914). Las condiciones y los límites del "progreso" liberal en los Territorios Nacionales. En P. Navarro Floria (Coord.), Paisajes del progreso: la resignificación de la Patagonia Norte, 1880-1916 (pp. 235-295). Neuquén: Universidad Nacional del Comahue.

Navarro Floria, P. (2008). El proceso de construcción social de la región del Nahuel Huapi en la práctica simbólica y material de Exequiel Bustillo (1934-1944). Pilquen, 10(1), 1-14. Recuperado de http://revele.uncoma.edu.ar/htdoc/ revele/index.php/Sociales/article/view/2065/58559

Papalia, M. (2012). Construcción de demandas políticas de comunidades Mbyá guaraníes en contextos de conservación de la naturaleza. Cuadernos de Antropología Social, 36, 119-150. Recuperado de http://revistascientificas.filo.uba.ar/index.php/CAS/article/ view/1354/1304

Pérez, P. y Delrio, W. (2019). Cambios y continuidades 
en las (des)territorializaciones estatales en Río Negro (1878-1955). En L. Kropff, P. Pérez, L. Cañuqueo y J. Wallace (Comps.), La tierra de los otros. La dimensión territorial del genocidio indígena en Río Negro y sus efectos en el presente (pp. 31-69). Viedma: Editorial Universidad Nacional de Río Negro.

Prieto, A. (2015). Para comprender a Formosa. Una aproximación a la historia provincial. 1879-1976. Buenos Aires: Editorial Dunken.

Redford, K. (1991). The Ecologically Noble Savage. Orion Nature Quarterly, 9(3), 24-29.

Restrepo, E. (2004). Esencialismo étnico y movilización política: tensiones en las relaciones entre saber y poder. En O. Barbary y F. Urrea (Eds.), Gente negra en Colombia. Dinámicas sociopolíticas en Cali y el Pacífico (pp. 227244). Medellín: Lealón.

Río Negro (25 de noviembre de 2017). Tras el choque con fuerzas federales hay un muerto en Villa Mascardi. Río Negro. Recuperado de https://www.rionegro.com. ar/bariloche/tras-el-choque-con-fuerzas-federales-hayun-muerto-en-villa-mascardi-FY3980700

Ruffini, M. (2005). Peronismo, territorios nacionales y ciudadanía política. Algunas reflexiones en torno a la provincialización. Avances del Cesor, V(5), 132-148.
Sapkus, S. (2014). Cambio agrario y reconfiguración de las relaciones sociales en la provincia de Formosa. Publicar, XII(16), 103-120. Recuperado de http://ppct.caicyt.gov. ar/index.php/publicar/article/view/4638/5071

Schmidt, M. (2013). Crónicas de un (des)ordenamiento territorial. Disputas por el territorio, modelos de desarrollo y mercantilización de la naturaleza en el este salteño (Tesis de doctorado). Buenos Aires: Facultad de Ciencias Sociales, Universidad de Buenos Aires - Teseo. Recuperado de https://www.teseopress.com/cronicas/

Trentini, F. (2015). Pueblos indígenas y áreas protegidas: procesos de construcción de identidades y territorialidades en el co-manejo del Parque Nacional Nahuel Huapi (Tesis de doctorado no publicada). Facultad de Filosofía y Letras, Universidad de Buenos Aires, Buenos Aires.

Trentini, F. (2016). Procesos de construcción de la diferencia cultural en el co-manejo del Parque Nacional Nahuel Huapi. Revista de Estudios Sociales, 55, 32-44. doi: https://doi.org/10.7440/res55.2016.02

Ulloa, A. (2001). El nativo ecológico: Movimientos indígenas y medio ambiente en Colombia. En M. Archila y M. Pardo (Eds.), Movimientos sociales, Estado y democracia en Colombia (pp. 286-320). Bogotá: ICANH y Centro de Estudios Sociales, Universidad Nacional de Colombia. 